\title{
Identification of $\gamma$-Irradiated Spices by Electron Spin Resonance (ESR) Spectrometry
}

\author{
(Received May 9, 1990)
}

\author{
Sadao UchiY Ama*, Yoko Kawamura* and Yukio SAIto* \\ (*National Institute of Hygienic Sciences: 1-18-1, Kamiyoga, Setagaya-ku, Tokyo, Japan)
}

\begin{abstract}
The electron spin resonance (ESR) spectrometry spectra of white (WP), black (BP) and red (Capsicum annuum L. var. frutescerns L., RP) peppers each had a principal signal with a $g$-value of 2.0043 , and the intensities of the principal signals were increased not only by $\gamma$-irradiation but also by heating. Irradiated RP also showed a minor signal $-30 \mathrm{G}$ from the principal one, and the intensity of the minor signal increased linearly with increasing dose from 10 to $50 \mathrm{kGy}$. Since the minor signal was observed in RP irradiated at $10 \mathrm{kGy}$ and stored for one year, but did not appear either after heating or after exposure to this signal is unique to $\gamma$-irradiated RP and should therefore be useful for the identification of $\gamma$-irradiated spices of Capsicum genus, such as paprika and chili pepper. The computer simulation of the ESR spectra suggested that the minor signal should be assigned to methyl radical and the principal signal mainly to a combination of phenoxyl and peroxyl radicals. Such minor signals were found in $\gamma$-irradiated allspice and cinnamon among 10 kinds of other spices.
\end{abstract}

Key words: identification of irradiated spices; electron spin resonance (ESR) spectrometry; irradiated spices; irradiated foods; white pepper; black pepper; red pepper; Capsicum annuum $\mathrm{L}$. var. frutescens $\mathrm{L}$.

\section{Introduction}

The $\gamma$-irradiation of various foods is growing in popularity as a substitute for the use of post harvest pesticides, and research on the identification of irradiated food is required internationally in order to ensure correct labelling of irriated foods in the market, and also to allow testing for excessive irradiation of foods ${ }^{1)}{ }^{1)}$. . In the United States of America, the irradiation of spices at a dose not exceeding $30 \mathrm{kGy}$ has been permitted for the purpose of pasteurization and insect disinfestation since $1986^{3}$. In Japan, the $\gamma$-irradiation of food is permitted only for inhibition of the sprouting of potato. Identification methods for other foods, such as irradiated spices, are still needed for the testing of imported foods.

Identification methods for irradiated foods have been investigated from the standpoint of changes of food constituents ${ }^{4)}$ ), biological and physical techniques ${ }^{6) \sim 10)}$, suspension of germination $^{\left.11 \sim 1^{3}\right)}$, radical formation ${ }^{14 \sim \sim 16)}$ and so on, but further internationally co-ordinated re- search is needed.

This paper deals with the identification of $\gamma$-irradiated peppers, including white (WP), black (BP) and red (RP, Togarashi in Japanese, Capsicum annuum L. var. frutescens L.) peppers, by radical measurement using electron spin resonance (ESR) spectrometry, and especially with a detailed study on a specific identification method of $\boldsymbol{\gamma}$-irradiated RP.

In the course of this work, however, our attention was drawn to a report by Wieser and Regulla $^{15)}$, who had discovered a similar specific signal in irradiated paprika (Capsicum annuun $\mathrm{L}$. var. longum SENDT) by using a phase sensitive ESR measurement technique. A detailed report of the measurement technique has not been published yet. We have carried out detailed research on $\mathrm{RP}$, which is a different variety from paprika and contains a larger amount of capsicin, by means of an ordinary ESR method, and the results are presented here. 
A

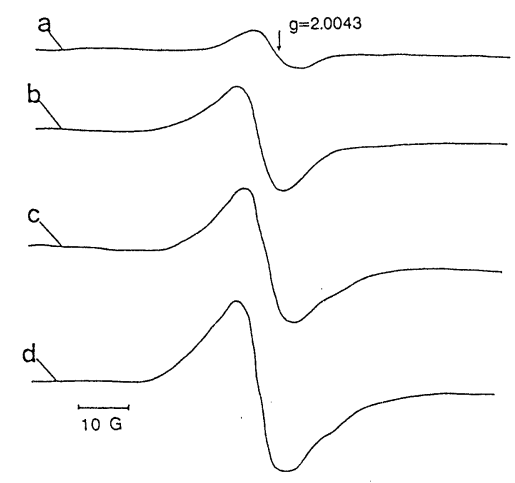

B

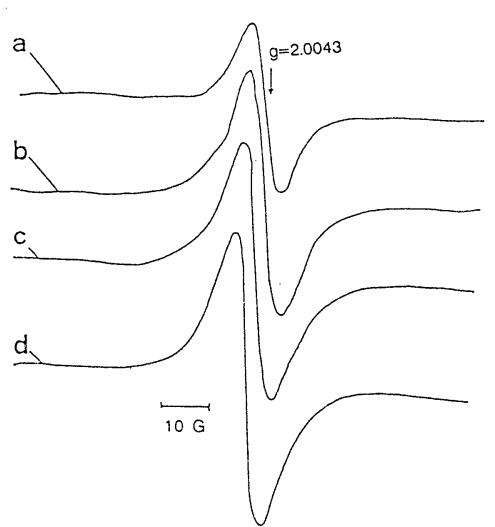

Fig. 1. ESR spectra of radicals produced in white pepper (WP) and black pepper (BP) by $\gamma$-irradiation

A: spectra of WP; B: spectra of BP; a: intact; b: irradiated at $10 \mathrm{kGy}$; c: irradiated at 30 $\mathrm{kGy}$; d: irradiated at $50 \mathrm{kGy}$. ESR spectra of WP and BP were measured at amplitudes of 790 and 125 , respectively.

\section{Experimental}

\section{Spices}

Spices used in the experiment were mainly white pepper (WP) (Piper nigrum L., without rind) produced in Malaysia, black pepper (BP) (Piper nigrum L., with rind) produced in Malaysia and red pepper (RP) (Capsicum annuum L. var. frutescens L.) produced in China. These powdered products (60 mesh) were obtained through the courtesy of Yasuma Co., Ltd. and the company guaranteed that they had not been irradiated. Other spices were purchased in the market in Japan. Bulky samples were cut into small pieces of less than $1 \times 1 \mathrm{~mm}$ in size.

Water contents of the above three peppers were calculated from the initial weight minus the constant weight when they were dried under diphosphorus pentoxide $\left(\mathrm{P}_{2} \mathrm{O}_{5}\right)$ in a desiccator for 60 days at room temperature $\left(22^{\circ} \mathrm{C}\right)$.

\section{$\gamma$-Irradiation}

Spices $(5 \mathrm{~g})$ in a glass test tube sealed with a glass stopper were irradiated in the range of 10 to $50 \mathrm{kGy}$ at an absorbed dose rate of $10 \mathrm{kGy} / \mathrm{hr}$ with a $\gamma$-irradiator $\left({ }^{60} \mathrm{Co}\right.$ source $)$ at the Japan Atomic Energy Research Institute (Takasaki City). The irradiated spices in the test tube were stored at room temperature in the dark.

\section{Heating and photo-exposure}

The peppers $(5 \mathrm{~g})$ in a glass test tube were heated at $100^{\circ} \mathrm{C}$ for one hour in an electric dryer and then stored at room temperature $\left(22^{\circ} \mathrm{C}\right)$ in a desiccator. RP (200 $\mathrm{mg}$ ) in a measurement tube for ESR was irradiated for one hour with a JEOL Photoirradiator Model ES-UV 10X using a xenon lamp $(31,000 \mathrm{~lx})$ placed $70 \mathrm{~cm}$ from the tube. RP (5 g) in a Petri dish was exposed to sunlight for one month from May to June.

\section{Measurement of ESR}

Spices $(200 \mathrm{mg})$ irradiated or not irradiated were placed in ESR-measurement tubes (outside diameter, $5 \mathrm{~mm}$; total length, $270 \mathrm{~mm}$; length of measurement part made of quartz, $100 \mathrm{~mm}$ ) and ESR measurements were carried out with a JEOL electron spin resonance spectrometer, Model TES-FE2XG, under the following conditions: field, $3,369 \pm 50 \mathrm{G}$; sweep time $2 \mathrm{~min}$; Mode, 6.3; amplitude, $1 \times 100 \sim 1 \times 1,000$; power, $1 \mathrm{~mW}$; temperature, $23^{\circ} \mathrm{C}$. Measurement of radical spin numbers, $g$-values and the simulation analyses of ESR spectra were performed with a JEOL ESR-data treatment system, Model ESPRIT-330.

Radical relative intensities of peppers were represented as the length from the top of signal to the bottom. Numbers of radical spins were 


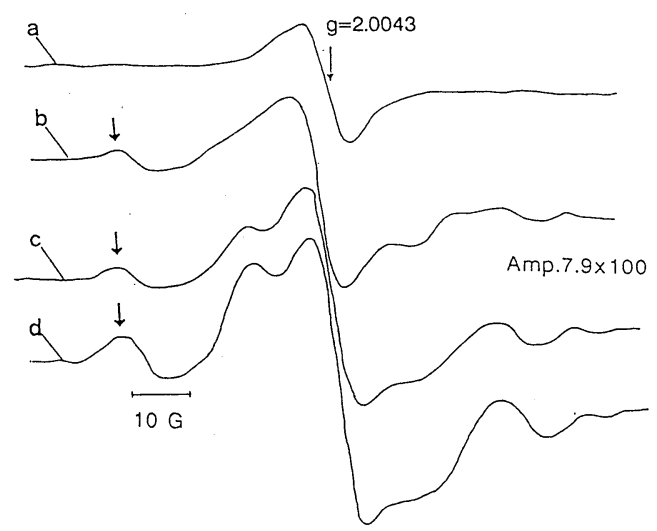

Fig. 2. ESR spectra of radicals produced in red pepper (RP) by $\boldsymbol{\gamma}$-irradiation a: intact; B: irradiated at $10 \mathrm{kGy}$; c: irradiated at $30 \mathrm{kGy}$; D: irradiated at $50 \mathrm{kGy}$. The arrows show the minor signal.

calculated by use of 2,2,6,6-tetramethylpiperidine- $N$-oxyl (TEMPOL) in benzene $\left(10^{-6} M\right)$ as a spin standard and the $g$-value was calculated by use of $\mathrm{MnO}$ as a $g$-value standard.

\section{Results}

\section{$\gamma$-Irradiation of three kinds of peppers}

The $\gamma$-irradiation of three kinds of peppers, white (WP), black (BP) and red pepper (RP), in the dose range of $10 \sim 50 \mathrm{kGy}$ resulted in radical generation. Irradiated WP showed a principal signal with a g-value of 2.0043 in the ESR spectrum and the signal intensity increased with increasing absorption dose, as shown in Fig. 1A. Intact WP, however showed the same signal as that of irradiated WP, though the signal intensity was low. Irradiated BP showed the same principal signal with the $g$-value of 2.0043 as that for irradiated WP, as shown in Fig. 1B, and the signal intensity increased with increasing dose. Intact BP showed the same signal as that of intact WP though the signal intensity was higher than that for intact WP.

On the other hand, irradiated RP had a different signal pattern from those of the above two peppers, as shown in Fig. 2. The principal signal with a $g$-value of 2.0043 and the minor signal (see arrow in the figure) in the low field $30 \mathrm{G}$ from the principal signal appeared in the ESR spectrum of irradiated RP. Both the signal intensities increased with increasing dose. The

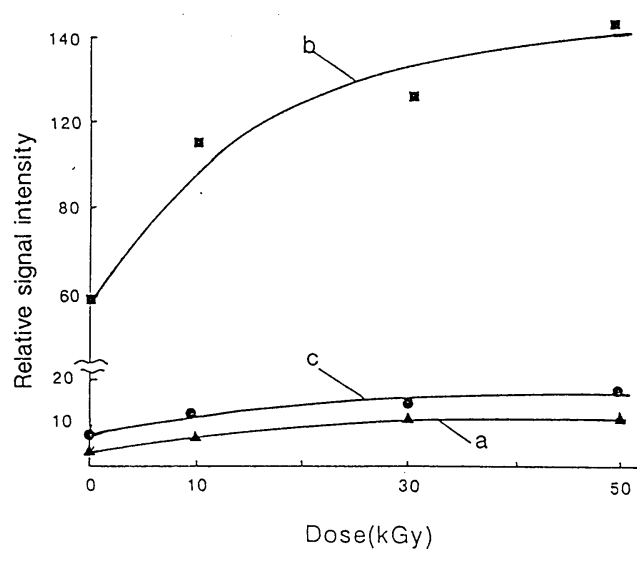

Fig. 3. Radical production in white pepper (WP), black pepper (BP) and red pepper (RP) by $\gamma$-irradiation

a: WP; b: BP; c: the principal signal in RP. The relative signal intensities of the principal signal with $g=2.0043$ were measured at the amplitude of 790 .

principal signal could be found in the spectrum of intact RP, but the minor one could not. The minor signals were also found in $\gamma$-irradiated products of paprika (two products), RP (two other products), chilli pepper (partially containing RP, a product), and ICHIMI TOGARASHI (partially containing RP, three products). Figure 3 shows the relationship between $\gamma$ absorbed dose and relative signal intensity of the principal signal (g-value of 2.0043) in the three kinds of peppers; the intensity of BP was the highest, and was approximately 10 times as high as those of the others at each dose. However, the signal intensity of intact BP (control) was also the highest among those of the intact peppers.

Water contents in WP, BP and RP were $9.83 \%$, $6.93 \%$ and $5.33 \%$, respectively. It therefore seemed clear that the radical production in these peppers was independent of water content, because there was no correlation between the signal intensity of generated radical in each pepper and water content.

\section{Radical production by heating}

Radical productions in heated peppers were compared with those in the intact peppers as shown in Fig. 4. The signal intensities ( $g$-value 


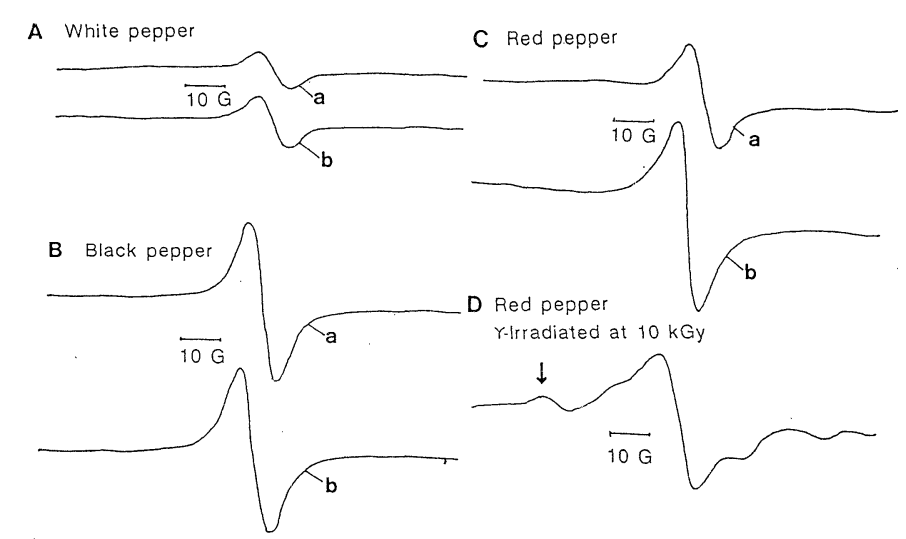

Fig. 4. ESR spectra of the three kinds of peppers heated at $100^{\circ} \mathrm{C}$ for one hour a: intact; b: heated. The arrow in spectrum D shows the minor signal.

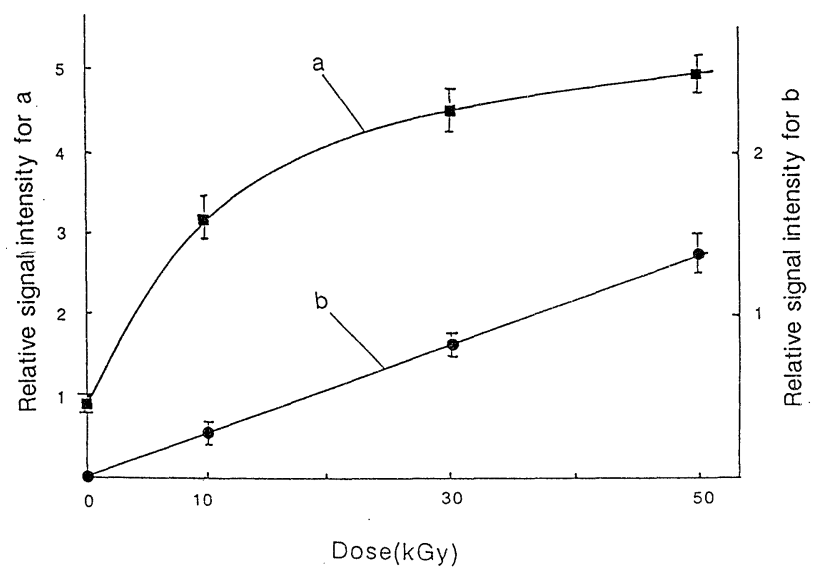

Fig. 5. Relationship between $\gamma$-absorption dose and radical production in red pepper (RP) a: the principal signal in PR; b: the minor signal in RP

The relative signal intensities in RP were measured at the amplitude of 100 at 17 days after $\gamma$-irradiation. Values are means \pm S.D. for four trials.

$=2.0043$ ) of $\mathrm{WP}$ and $\mathrm{BP}$ heated at $100^{\circ} \mathrm{C}$ for one hour were increased by $50 \%$ and $10 \%$ or more, respectively. On the other hand, the intensity of the principal signal of heated RP was also increased two-fold, but the minor signal produced by $\gamma$-irradiation did not appear (see Fig. 4D).

\section{Appearance of the minor signal in $R P$}

Since the minor signal appeared specifically in $\mathrm{RP}$ after $\gamma$-irradiation, the properties of the minor signal in irradiated RP were investigated in detail in order to develop an identification method specific to $\gamma$-irradiated spices. The signal intensities of the principal and minor signals both increased dose-dependently, as shown in Fig. 5. Although the minor signal intensity was about one-sixth as high as the principal one on irradiation at the dose of 10 $\mathrm{kGy}$, the dose-response curve was linear and passed through the origin. The same tendency was observed when the radical spin numbers were expressed in the ordinate instead of signal intensity.

The appearance of the minor signal in RP upon light-irradiation was examined under sunlight and artificial light (Xe-lamp). When RP was exposed to sunlight for one month from May to June, the intensity of the principal signal was not affected and the minor one did not appear, as shown in Fig. 6. Even when RP was exposed 
at room temperature $\left(22^{\circ} \mathrm{C}\right)$ for one hour to a Xe-lamp, which usually effectively promotes photolysis, the minor signal was not observed. This means that the minor signal is peculiar to $\gamma$-irradiated RP.

\section{Computer simulation of ESR signals}

Radical identification in $\gamma$-irradiated RP was performed by a computer-simulation method. Several trials of simulation showed that the spectrum of irradiated RP was identical with that of a mixture of three types of radicals from methyl, phenoxyl and peroxyl groups. Table 1 summarizes the factors used for the computer

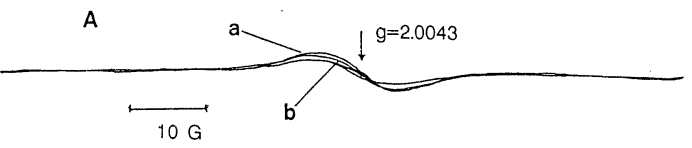

B

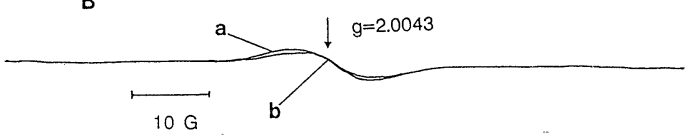

Fig. 6. Radical production of red pepper by exposure to light of a Xe-lamp or to sunlight A: exposed to a Xe-lamp for one hour; B: exposed to sunlight for one month; a: intact; b: exposed to light.

These spectra were measured at the amplitude of 100 . simulation. Radical numbers 1,2 and 3 in the table stand for radicals from methyl, phenoxyl and peroxyl groups, respectively. Each peak was expressed as a Lorentz curve. Spectral range and data length stand for the range of signal and scale on a chart, respectively. In Fig. 7 , the simulated signal of the methyl radical is shown by spectrum $A$. The combined signal of methyl and phenoxyl radicals is shown by spectrum $\mathrm{B}$, and finally the combined signal of methyl, phenoxyl and peroxyl radicals is shown by spectrum $C$, which is very similar to that (D) of RP irradiated at $30 \mathrm{kGy}$. It is concluded that the minor signal is attributable to the methyl radical and the principal signal $(g=2.0043)$ is mainly attributable to a combination of pheno$\mathrm{xyl}$ and peroxyl radicals.

\section{Life span of radicals}

The radical life span of irradiated RP was examined by monitoring the radical intensity for one year at room temperature $\left(22^{\circ} \mathrm{C}\right)$. Figure 8 shows the decay curves of both the principal and the minor signals. The intensities of the principal signal run at an amplitude of 100 declined rapidly immediately after irradiation and slowly thereafter (Fig. 8A). The intensities of the minor signal were measured at higher sensitivity with an amplitude of 790 . The pattern of decline was similar to that of the principal signal. However, the minor signal was detectable (and was useful for the detection of ir-

Table 1. Factors for Computer-simulation of Radical Assignment in $\gamma$-Irradiated Red Pepper

\begin{tabular}{|c|c|c|c|c|}
\hline \multicolumn{2}{|c|}{ Radical number } & 1 & 2 & 3 \\
\hline \multicolumn{2}{|c|}{ Line width $[\mathrm{mT}]$} & 0.7000 & 0.9200 & 1.5000 \\
\hline \multicolumn{2}{|c|}{ Intensity } & 30 & 75 & 90 \\
\hline \multicolumn{2}{|c|}{ Lorentz (\%) } & 100 & 100 & 100 \\
\hline \multicolumn{2}{|c|}{ Gauss (\%) } & 0 & 0 & 0 \\
\hline \multicolumn{2}{|c|}{ Center field $[\mathrm{mT}]$} & 337.00 & 336.90 & 336.74 \\
\hline \multicolumn{2}{|c|}{$g$-Value } & 2.0034 & 2.0040 & 2.0050 \\
\hline \multirow{2}{*}{\multicolumn{2}{|c|}{ Spectral range }} & 333.49 & 336.90 & 336.74 \\
\hline & & 340.51 & 336.90 & 336.74 \\
\hline Nuclear & Spin & 0.5 & 0.5 & 0.5 \\
\hline \multirow[t]{2}{*}{1} & Number & 3 & 1 & 1 \\
\hline & $\mathrm{Hfcc}[\mathrm{mT}]$ & 2.340 & 0.000 & 0.000 \\
\hline
\end{tabular}

Frequency $[\mathrm{MHz}] 9450.000$

Data length 2000 


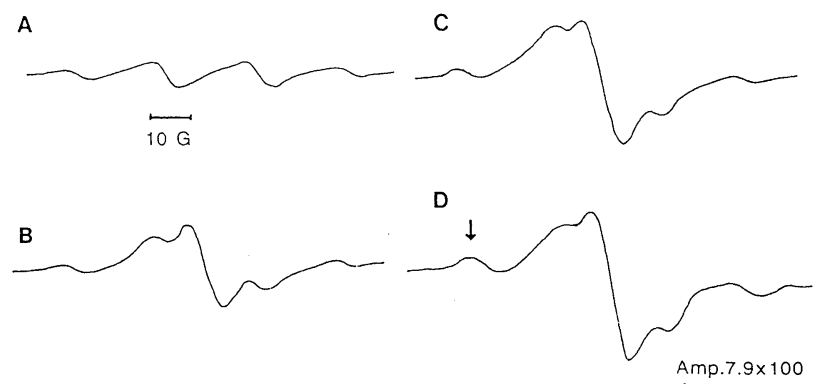

Fig. 7. Identification of radicals in $\gamma$-irradiated red pepper (RP) by a computer-simulation method

A: a simulated spectrum of methyl radical; B: a simulated spectrum of combined methyl and phenoxyl radicals; C: a simulated spectrum of combined methyl, phenoxyl and peroxyl radicals; D: a spectrum of $\mathrm{RP} \gamma$-irradiated at $30 \mathrm{kGy}$

The arrow in spectrum $\mathrm{D}$ shows the minor signal.
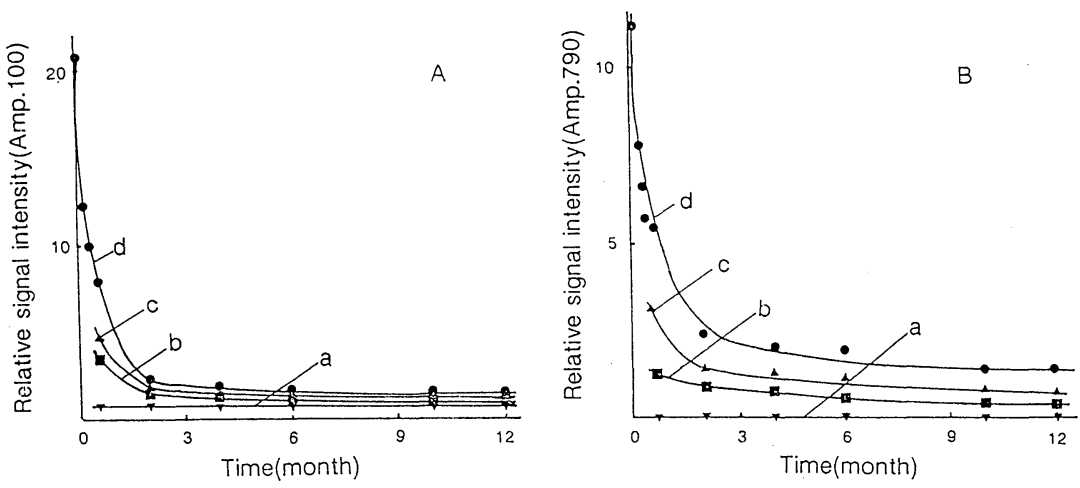

Fig. 8. Decay curves of radicals produced in red pepper by $\gamma$-irradiation

A: the principal signal intensity $(g=2.0043)$; B: the minor signal intensity; $a$ : intact; b: irradiated at $10 \mathrm{kGy}$; : irradiated at $30 \mathrm{kGy}$; d: irradiated at $50 \mathrm{kGy}$

radiated $\mathrm{RP}$ ) even after one year.

The radical spin number for the principal in $\mathrm{RP}$ irradiated with a dose of $50 \mathrm{kGy}$ and stored for one year was $1.05 \times 10^{16} / \mathrm{g}$ and that for the minor signal was $3.18 \times 10^{14} / \mathrm{g}$. The spin number for the principal signal in intact RP was $1.63 \times 10^{15} / \mathrm{g}$. The radical spin numbers for the principal signals in WP and BP irradiated at 50 $\mathrm{kGy}$ and stored for one year were $1.58 \times 10^{16} / \mathrm{g}$ and $6.05 \times 10^{16} / \mathrm{g}$, respectively, and those in intact WP and BP were $1.83 \times 10^{15} / \mathrm{g}$ and $3.05 \times$ $10^{16} / \mathrm{g}$, respectively.

The minor signal could be detected after longer than one year by increasing the sensitivity further. In Fig. 9, spectrum $A$ is that of intact $\mathrm{RP}$ and spectrum $\mathrm{B}$ is that of $\mathrm{RP}$ irradiated at $10 \mathrm{kGy}$. The minor signal in the spectrum became obscure one year after $\gamma$ - irradiation but was confirmed clearly by raising the amplitude from 790 to 1000 , as shown in Fig. 9C-a and C-b.

Radical production by $r$-irradiation of other spices

The ESR method was applied to ten other kinds of spices to examine its availability as an identifiction method for irradiated spices. Almost all of the irradiated spices showed the principal signals. The spices having the minor signal peculiar to $\gamma$-irradiation were cinnamon and allspice, besides the Capsicum products such as RP, as shown in Fig.10.

\section{Discussion}

A method for the identification of irradiated WP, BP and RP is required to confirm the correctness of labelling of irradiated foods in the 

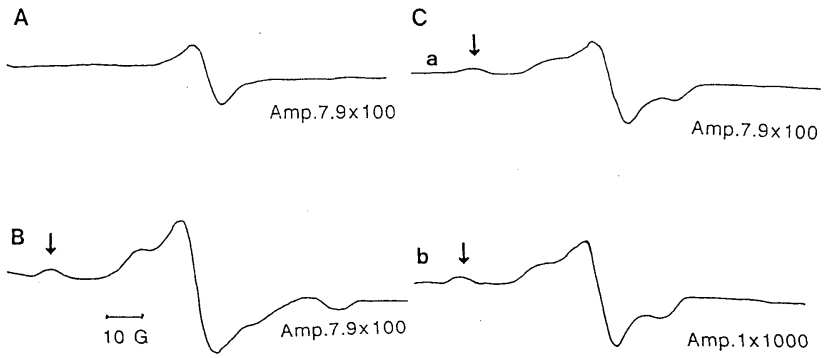

Fig. 9. Comparison of the ESR spectrum of red pepper measured immediately after $\gamma$-irradiation $(10 \mathrm{kGy})$ with that measured after one year

A: intact; B: measured immediately after $\gamma$-irradiation; $\mathrm{C}$ : measured in one year after $\gamma$-irradiation; C-a: measured at the amplitude of 790; C-b: measured at the amplitude of 1000

The arrows show the minor signals.
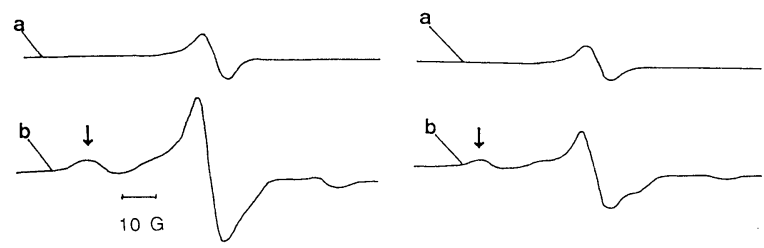

Fig. 10. ESR spectra of other $\gamma$-irradiated spices a: intact; b: irradiated at $50 \mathrm{kGy}$ These spectra were measured at the amplitude of 100 .

The arrows show sub-signals corresponding to the minor signal in red pepper.

market, and also to test imported foods, because these peppers are imported in comparatively large quantity into Japan, where use of irradiated spices is prohibited. Radical formation in $\gamma$-irradiated food is a well-known phenomenon ${ }^{17), 18)}$ which might be applicable to the identification of irradiated spices.

It was found that $\gamma$-irradiation with absorbed doses from 10 to $50 \mathrm{kGy}$ formed many radicals corresponding to the principal signal with the $g$-value of 2.0043, as shown in Figs. 1 and 2. The principal signal was also found in intact peppers. Radicals in the intact peppers would be produced merely by energy-providing processes such as heating and light-exposure in the process of manufacturing. Among these peppers, only irradiated RP showed the minor signal $-30 \mathrm{G}$ from the principal one, as shown in Fig. 2. Wieser and Regulla ${ }^{15)}$ found a signal similar to the minor one in irradiated paprika, which is a different variety of Capsicum genus from RP used in this experiment, by ESR measurement using a phase-sensitive technique. Although details of their technique are not yet available, we have found such a minor signal in RP irradiated with more than $10 \mathrm{kGy}$ by ordinary ESR measurement and showed that it was utilizable for specific identification of irradiated RP. On the other hand, the principal signal was enhanced not only by irradiation but also by heating of the three kinds of peppers, as shown in Fig. 4. The principal signal, therefore, is not applicable to specific identification of irradiated peppers. However, the minor signal in irradiated RP was not seen after heating or after light-irradiation with a Xe-lamp or exposure to sunlight (Fig. 6). It was therefore found that the minor signal was unique to irradiated RP and applicable to the identification of irradiated RP. Since the minor signal could be observed in 
other analogous irradiated spices such as other $\mathrm{RP}$ products, paprika and chilli pepper, the method might be generally applicable to irradiated spices of Capsicum genus.

The radicals corresponding to the principal signal may be generated by energy inputs such as heating and $\gamma$-irradiation and almost all spices may contain substances sensitive to these reactions. Intact BP itself showed higher signal intensity than that of WP, even though both peppers were said to have been produced from the same fruit of Piper nigrum L. This may be attributed to the fact that BP contains the whole fruit rind but WP contains it only partially. The dry rind may be particularly liable to form radicals by heating and $\gamma$-irradiation. RP and analogous peppers appear to contain substances sensitive only to $\gamma$-irradiation, though their identity is unknown at present.

The relationship between a dose absorbed and relative signal intensity was convex for the principal signal but linear for the minor signal (Fig. 5). Even when the radical spin number was expressed as the intensity, the same tendency was seen in both the principal and minor signals. There is therefore a possibility that an identification method using the minor signal may be utilized for quantitative analysis of irradiated RP. The method would be useful in detection of RP irradiated at $5 \mathrm{kGy}$.

From the results in the computer-simulation of the ESR spectrum, it was apparent that the principal signal in irradiated RP would be derived mainly from a combination of phenoxyl and peroxyl radicals, and the minor signal from methyl radical (Fig. 7). The signal with $\mathrm{g}=$ 2.0043 in irradiated WP and BP is probably derived from the same combination of phenoxyl and peroxyl radicals as that in irradiated RP. The reason why the methyl radical is produced in RP by $\gamma$-irradiation and is stabilized in the RP for a long time is unknown but may be related to the existence of high-molecular matrix trapping radicals, such as the $\cdot \mathrm{CO}_{2}^{-}$radicals in hydroxyapatite of irradiated bone ${ }^{18)}$ or $\cdot \mathrm{C}$ radicals in irradiated protein ${ }^{17)}$.

The relative signal intensities of the principal and the minor signals in the RP decayed sharply after $\gamma$-irradiation and slowly thereafter (Fig. 8). The decay of the signal intensity is disadvanta- geous from the viewpoint of a quantiative analysis method for irradiated food. The minor signal in RP stored for one year after $\gamma$-irradiation was indistinct, as shown in Fig. $9 \mathrm{C}$-a, but a distinct signal could be recovered by measuring the ESR spectrum at higher amplitude. Thus, RP irradiated at $10 \mathrm{kGy}$ and stored for one year was distinguishable from intact RP. By means of the method, the identification of RP irradiated at $10 \mathrm{kGy}$ and more was possible but it was not possible to determine the absorbed dose in the pepper because of the decay of relative signal intensity during storage of the pepper, especially the sharp decay within one month after $\gamma$ irradiation. There is, however, a possibility that the dose may be calculated if the storage period after irradiation is known or can be estimated.

With other irradiated spices, signals similar to the minor signal in irradiated RP were found in allspice and cinnamon among 10 different kinds of spices (Fig. 10). This ESR method using the unique minor signal may be applicable to the identification of these irradiated spices in addition to spices in Capsicum genus.

Water content in WP was the highest among the three peppers but the signal intensity or the total number of radical spins produced by $\gamma$ irradiation was the highest in BP. From the results of this experiment, it appears that there is no relation between radical production and water content under $10 \%$ in the three peppers. However, radicals are generally liable to be quenched by moisture, and the production of radicals did vary with water content in paprika $^{16)}$ reportedly. In this experiment, although irradiated RP was stored at room temperature of $22^{\circ} \mathrm{C}$, the minor signal was detectable even after one year. However, the temperature of storage is expected to affect radical decay. Further studies on the effects of water contents and storage temperature may be necessary for each kind of spice.

Although the principal signals shown in the three peppers were increased by both heating and $\gamma$-irradiation, there is still a possibility that the signal may be applicable to the identification of irradiated spices, if information on the processing conditions of the spices, such as date of irradiation, temperature in the processing stage and so on, is available. 


\section{Acknowledgments}

The authors are grateful to Mr. Tamiji Sato for assistance in operating ESR, to Dr. Hitoshi Ito, Dr. Tamikazu Kume and Dr. Isao Isigaki of Japan Atomic Energy Research Institute for their cooperation in $\gamma$-irradiation, to Dr. Masahiro Kohno of JEOL Ltd. and his cowokers for their technical support of ESR computer-simulation, and to Dr. Makoto Kato of Yasuma Ltd. and coworkers for their kind supply of WP, BP and $\mathrm{RP}$ and their advice.

\section{References}

1) World Health Organization (WHO): "Food Irradiation, a technique for preserving and improving the safety of food", p. 33 55 (1988) WHO, Geneva, Switzerland.

2) Diehl, J. F.: “Safety of Irradiated Foods", p. 87 94 (1990) Marcel Dekker, Inc., New York.

3) Food and Drug Administration, Department of Health and Human Services: Federal Register, 51 (75), 21CFR part 179. 26, p. 13, 399 (April 18, 1986).

4) Uchiyama, S., Nagasima, K., Kawamura, Y. Saito, Y.: J. Food Hyg. Soc. Japan 29, 395 401 (1989).
5) Toyo'oka, T., Uchiyama, S., Saito, Y.: J. Agric. Food Chem. 37, 770 775(1989).

6) Delincée, H.: Institut fur Strahlen Hygiene (ISHHeft) 125, 248 265 (1988).

7) Heide, L., Bögl, K. W.: ibid. 125, 207 232 (1988).

8) Goksu-ogelman, H. Y., Regulla, D. F.: Nature 340, 23 (1989).

9) Sanderson, D. C. W., Slater, C., Cairns, K. J.: ibid. 340, 23 24 (1989).

10) Hayashi, T., Iwamoto, M., Kawashima, K.: Agric. Biol. Chem. 46, 905 912 (1982).

11) Uchiyama, S., Konno, S., Toyo'oka, T., Kawamura, Y., Saito, Y.: J. Food Hyg. Soc. Japan 30, 152 159 (1989).

12) Kawamura, Y., Uchiyama, S., Saito, Y.: J. Food Sci. 54, 379 382 (1989).

13) Kawamura, Y., Uchiyama, S., Saito, Y.: ibid. 54, $1,501 \sim 1,504$ (1989).

14) Dodd, N. J. F., Swallow, A. J. , Ley, F. J.: Radiat. Phys. Chem. 26, 451 453 (1985).

15) Wieser, A., Regulla, D. F.: Institut fur Strahlen Hygiene (ISH-Heft) 125, 155 161 (1988).

16) Beczner, J., Farkas, J., Watterich, A., Mailath, P. F., Kiss, I.: ibid. 125, 162 171 (1988).

17) Uchiyama, S., Uchiyama, M.: J. Food Sci. 44, 1,217 1,220 (1979).

18) Geoffroy, M., Tochon-Danguy, H. J.: Int. J. Radiat. Biol. 49, 621 (1985). 